\title{
Ultrasound-guided retrieval of lost intrauterine devices using very fine grasping forceps: a case series
}

\author{
Francesca Moro, ${ }^{1}$ Jure Knez, ${ }^{1}$ Katie Pateman, ${ }^{1}$ Grigorios Derdelis, ${ }^{1}$ \\ Xulin Foo, ${ }^{1}$ Davor Jurkovic ${ }^{2}$
}

${ }^{1}$ Clinical Research Fellow, Institute of Women's Health, University College London, London, UK

${ }^{2}$ Consultant Gynaecologist, Institute of Women's Health, University College London, London, UK

\section{Correspondence to Dr Davor Jurkovic, Gynaecology Diagnostic and Outpatient Treatment Unit, Elizabeth Garrett Anderson Wing, University College Hospital, 235 Euston Road, London NW1 2BU, UK; davor.jurkovic@nhs.net}

Received 8 September 2014 Revised 3 December 2014 Accepted 1 January 2015 Published Online First 3 February 2015

\begin{abstract}
Aim To assess the efficacy of a novel ultrasoundguided procedure for the retrieval of intrauterine contraceptive devices (IUDs) when the threads are not visible at the external cervical os ('lost threads'). Methods This was a prospective cohort study of consecutive women referred for ultrasound examination because of lost IUD threads. The procedures were performed under local anaesthesia in the outpatient setting. After injection of local anaesthetic, the anterior cervical lip was grasped with a vulsellum forceps. A $5 \mathrm{Fr}$ hysteroscopy grasping forceps was introduced transcervically into the uterine cavity under continuous transabdominal ultrasound guidance. The IUD was then grasped and removed from the uterus. Patients' demographic data, gynaecological history, ultrasound findings, duration of procedure, success rate and pain score were recorded.
\end{abstract}

Results Twenty-three consecutive women were included in the study. Ultrasound examination showed an IUD correctly sited in the centre of the uterine cavity in 20/23 (87\%), in 2/23 (9\%) it was partially embedded in the myometrium and in $1 / 23$ (4\%) the IUD was partially sited in the cervical canal. In 8/23 (35\%) women the IUD threads were not visible on ultrasound scan. Removal of the IUD was successful in 22/23 (96\%) cases with a median operating time of 3 (interquartile range 1.25-4.75) minutes. 15/23 (65\%) women experienced no or minimal pain (pain score $\leq 3$ ), 4/23 (17\%) reported moderate pain (pain score 4-6) and 4/23 (17\%) described the pain as severe (pain score 7-10). No complications were recorded during or immediately after the procedure.

Conclusions Ultrasound-guided retrieval of lost IUDs using fine hysteroscopy grasping forceps is a highly successful technique and is well tolerated by women.

\section{INTRODUCTION}

To cite: Moro F, Knez J, Pateman K, et al. J Fam Plann Reprod Health Care 2015;41:205-209.
Intrauterine devices (IUDs) are among the most commonly used forms of reversible contraception worldwide. ${ }^{1}$ Nowadays their use extends beyond

\section{Key message points}

- Ultrasound-guided retrieval is a novel approach to 'lost' intrauterine device removal.

- Our initial results show that ultrasoundguided retrieval it is more successful and less painful when compared to currently available alternative methods.

contraception, since the $52 \mathrm{mg}$ progestogen-releasing IUD is often used as the treatment of choice for heavy periods and for endometrial protection in hormone replacement therapy. ${ }^{2} 3$

Removal of an IUD is usually a simple procedure. It involves grasping the threads with a forceps and applying gentle traction to extract it through the cervical canal. ${ }^{4}$ In $5-18 \%$ of women, however, the threads cannot be visualised on speculum examination; this occurs either because the threads retract into the cervical canal or uterine cavity, or they become wrapped around the body of the IUD. They can also detach from the body of the IUD and be expelled spontaneously during menstrual periods. ${ }^{4-7}$ In these women further investigations are required to determine the location of the IUD. ${ }^{48}$

A variety of instruments have been utilised to retrieve IUDs with missing threads from the uterine cavity. They include artery forceps (e.g. Spencer Wells) to grasp the threads in the cervical canal, purposefully designed plastic IUD thread retrievers and extractor hooks, and purposefully designed forceps. Procedures using these devices are usually performed blindly with reported success rates of $37-59 \% .^{5}$ In cases where these 
procedures fail, women are usually referred for IUD removal under direct vision using hysteroscopy. Although this is a very successful procedure, it has to be performed by highly skilled operators, it cannot be used in pregnant women and it is comparatively costly. ${ }^{6} 9$

Ultrasound is used routinely for the assessment of women presenting with a wide range of gynaecological complaints and it also provides clear images of an IUD within the uterus. As a result ultrasound is often used to check the position of an IUD after its insertion. ${ }^{10}$ It is also useful for locating an IUD when the threads are not visible. ${ }^{8}$ Ultrasound-guided procedures are often used in gynaecology, particularly in the field of reproductive medicine and assisted reproduction. Ultrasound guidance may also be used to minimise the risk of uterine perforation and other complications during intrauterine gynaecological procedures. ${ }^{11}$ The aim of this study was to assess the feasibility, success rate and women's tolerance of ultrasound-guided retrieval of IUDs with lost threads from the uterus using fine hysteroscopy forceps.

\section{METHODS}

\section{Study population}

This was a prospective observational study that was conducted at the Department of Gynaecology, University College London Hospital, London, UK from March 2013 to March 2014.

We included consecutive women who were referred by their general practitioners or family planning doctors for removal of an IUD when the threads were not visible on speculum examination or when previous attempts to remove the IUD had failed.

Demographic data, clinical history, indications for IUD insertion and duration of use were all recorded.

The inclusion criteria were: IUD located inside the uterus on ultrasound examination and ability to tolerate vaginal speculum examination. A full urinary bladder was not required for the procedure. All the women gave written, informed consent after receiving information about all aspects of the procedure and the potential risks. Ultrasound-guided intrauterine procedures are part of routine clinical practice in our unit and ethical approval for the study was therefore not required.

\section{Interventions}

A transvaginal ultrasound examination was performed first (Voluson ${ }^{\mathrm{TM}}$ E8, GE Medical Systems, Milwaukee, WI, USA) in order to assess uterine morphology and position. The presence of any congenital uterine anomaly, fibroids or adenomyosis was recorded. The exact positions of the IUD and the threads were then ascertained, including any signs of the IUD being partially or completely embedded in the myometrium.

The ultrasound probe was then removed and a Cusco speculum was inserted. The cervix was examined visually for the presence of IUD threads and assessed for any signs of abnormality. It was then cleansed with an antiseptic solution and 1-2 $\mathrm{ml} \mathrm{1 \%}$ lidocaine solution was injected into the anterior lip. A vulsellum forceps was then applied in order to exert traction and correct uterine flexion during the procedure. A second operator used a transabdominal probe to provide continuous ultrasound guidance. The probe's position was such that it provided a longitudinal view of the uterus and the cervix at all times. Thus the hysteroscopy forceps could be visualised in its whole length when introduced into the uterine cavity through the cervix.

A $1.67 \mathrm{~mm}$ (5Fr) hysteroscopy grasping forceps [Karl Storz Endoscopy (UK), Ltd, Slough, UK] (Figure 1) was lubricated with gel containing lidocaine hydrochloride $2 \%$ and chlorhexidine gluconate $0.25 \%$ (Instillagel $^{\circledR}$, CliniMed Ltd, Loudwater, UK). The grasping forceps was then inserted into the cervical canal under continuous transabdominal ultrasound guidance. In women with ultrasound-visible threads an attempt was made to grasp them with the forceps and then to remove the IUD (Figure 2). In women with no visible threads, and in those women in whom the threads could not be grasped, the body of the IUD was held with the forceps and removal was attempted. In women with a rotated IUD and in those in whom traction on the body of the IUD was unsuccessful, an attempt was made to grasp a plastic arm of the IUD. Once the IUD was successfully grasped it was removed by applying continuous slow traction until the IUD was extracted through the external os. Antibiotic prophylaxis was not used routinely.

The duration and success of the procedure were recorded in each case. The level of pain the women

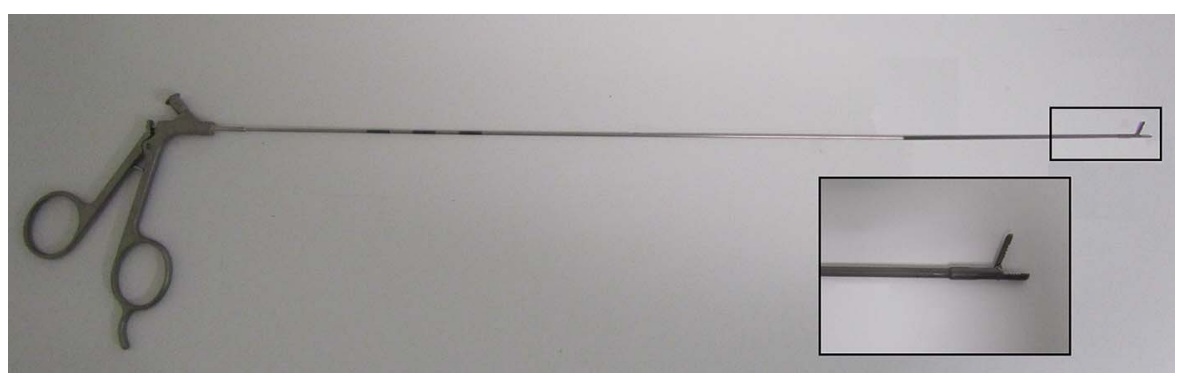

Figure 1 Photograph of $1.67 \mathrm{~mm}$ (5Fr) hysteroscopy grasping forceps with open jaws. 


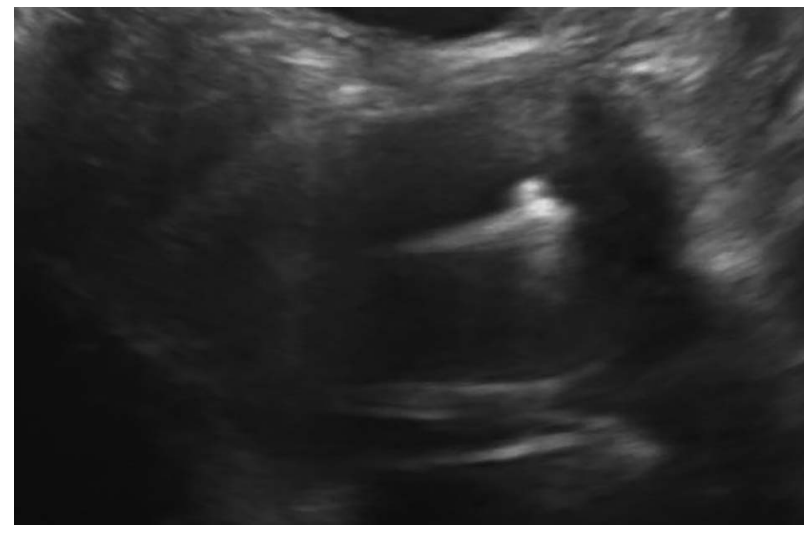

Figure 2 Longitudinal transabdominal ultrasound view of the uterus with inserted hysteroscopy forceps. The jaws of the instrument are opened before the intrauterine device is grasped and removed.

experienced was assessed 10-15 minutes after completion of the procedure using a visual analogue scale between 0 (no pain) to 10 (maximum pain). ${ }^{12}$

\section{Statistical analysis}

The primary outcome of the study was the success of ultrasound-guided retrieval of the missing IUD. The secondary outcome was the pain score. The SmirnovKolmogorov test was used to test for the normal distribution of data. Women's age, parity, duration since IUD insertion, pain score and duration of the procedure were not normally distributed and they were expressed as medians with interquartile ranges (IQRs). Proportions were expressed as percentages. A univariate logistic regression was performed using pain score (categorical) as the dependent variable and age, parity (categorical), type of IUD, time since insertion, uterine version, embedment into the myometrium and presence of fibroids as independent variables. A value of $p<0.05$ was taken as significant. All statistical analyses were carried out using SPSS V.19TM (SPSS Inc., Chicago, IL, USA).

\section{RESULTS}

Twenty-three consecutive women were included in the study. The indications for IUD removal are listed in Table 1. Demographic characteristics and relevant ultrasound data are listed in Table 2.

Table 1 Indications for intrauterine contraceptive device removal $(n=23)$

\begin{tabular}{lcc}
\hline Indication & $\mathrm{n}(\%)$ \\
\hline Routine replacement & $12(52)$ \\
Abnormal uterine bleeding & $3(13)$ \\
Abnormal uterine bleeding and pelvic pain & $2(9)$ \\
Desire for conception & 2 & $(9)$ \\
Contraception not required & 2 & $(9)$ \\
Pelvic pain & 1 & $(4)$ \\
Misplaced intrauterine device following insertion & 1 & $(5)$ \\
\hline
\end{tabular}

Table 2 Demographic characteristics and ultrasound features of the population at the time of intrauterine device removal

\begin{tabular}{|c|c|c|}
\hline Demographic characteristics/ultrasound features & $\mathrm{n}(\%$ & \\
\hline Age (median, IQR) & 413 & $7-49)$ \\
\hline Menstrual history & & \\
\hline Postmenopausal & 9 & (39) \\
\hline Amenorrhoea & 8 & (35) \\
\hline Luteal phase of menstrual cycle & 3 & (13) \\
\hline Proliferative phase of menstrual cycle & 2 & (9) \\
\hline Irregular vaginal bleeding & 1 & (4) \\
\hline Parity & & \\
\hline Nulliparous & 6 & (26) \\
\hline Parity (median, range) & 2 & $(1-4)$ \\
\hline Previous Caesarean section & 9 & (39) \\
\hline Uterine position & & \\
\hline Anteverted & 17 & $(74)$ \\
\hline Retroverted & 6 & (26) \\
\hline Uterine morphology & & \\
\hline Normal & 14 & $(60)$ \\
\hline Uterus enlarged by multiple IM/SS fibroids & 5 & $(22)$ \\
\hline Uterus with a single IM/SS fibroid & 2 & (9) \\
\hline Single SM fibroid & 1 & (4) \\
\hline Adenomyosis & 1 & (4) \\
\hline IUD type & & \\
\hline Mirena ${ }^{\circledR}$ IUS & 17 & (74) \\
\hline Copper IUD & 5 & $(22)$ \\
\hline Ring IUD & 1 & (4) \\
\hline IUD removal attempt before referral & 9 & (39) \\
\hline IUD position & & \\
\hline Correctly placed at the centre of the uterine cavity & 20 & $(87)$ \\
\hline Embedded in the myometrium & 2 & (9) \\
\hline Partially expelled into the cervical canal & 1 & (4) \\
\hline Threads position & & \\
\hline Retracted into the upper half of the cervical canal & 10 & $(43)$ \\
\hline Retracted into the lower half of the cervical canal & 6 & $(26)$ \\
\hline Not visualised & 7 & $(30)$ \\
\hline
\end{tabular}

*Values are $n(\%)$ unless stated otherwise.

$I M$, intramural; IQR, interquartile range; IUD, intrauterine device; IUS, intrauterine system; SM, submucous; SS, subserous.

Some 14/23 (61\%) women had used their IUD or intrauterine system (IUS) for contraception, 7/23 (30\%) for menorrhagia (IUS), 1/23 (4\%) for dysmenorrhoea (IUS) and 1/23 (4\%) as a part of hormone replacement therapy (IUS). The median duration between insertion and the removal procedure was 5 (range 1-15) years.

The threads were not visible in the cervical canal on scanning in $7 / 23(30 \%)$ of cases (Table 2).

The procedure was successful in 22/23 (96\%) of cases. In all women the grasping forceps was clearly visible and its position was continuously monitored on ultrasound.

Pain experienced during the procedure is presented in Table 3. The median pain score was 3 (IQR 0-5). Univariate analysis did not show any significant 
Table 3 Pain experienced during the removal of a lost intrauterine device

\begin{tabular}{ll}
\hline Pain score & $\mathrm{n}(\%)$ \\
\hline Painless (pain score 0) & $7(30)$ \\
Mild pain (pain score 1-3) & $8(35)$ \\
Moderate pain (pain score 4-6) & $4(17)$ \\
Severe pain (pain score 7-10) & $4(17)$ \\
\hline
\end{tabular}

association between women's age, parity, type of IUD, time since insertion, uterine position, embedment into the myometrium or presence of fibroids, and pain scores during the procedure $(p>0.05)$.

There were no intra- or post-operative complications and none of the women reported any clinical signs of infection after the procedure. In $7 / 23(30 \%)$ women a new IUD was inserted at the end of the procedure.

The procedure was unsuccessful due to inability to identify the external os and the cervical canal in one woman who had previously undergone a cone biopsy for treatment of cervical intraepithelial neoplasia. In her case, the scar tissue was later excised using a loop electrode under general anaesthesia to reveal the entrance to the cervical canal. The IUD was then removed using the same ultrasound-guided technique.

\section{DISCUSSION}

Our study has shown that our novel ultrasound guided method was highly successful for retrieving IUDs in women with missing threads. Our success rate of 96\% [95\% confidence interval (CI) 87-100] was significantly better than the findings of the randomised study by Bounds et al. ${ }^{5}$ who reported success rates of 53\% (95\% CI 40-65) with Emmett and 59\% (95\% CI 47-70) with Retrievette ${ }^{\circledR}$ retriever devices. However, our results were similar to the success rate of $94.7 \%$ (95\% CI 84.6-100.0) reported with the use of outpatient hysteroscopy. ${ }^{9}$ Another recent study assessed the efficacy of ultrasound-guided use of a bent Cook catheter or a crochet-type hook when the threads were not retrievable using alligator forceps. The reported success rate in this study was $87 \%$ (95\% CI 76-98). ${ }^{13}$

The main advantage of our technique is the use of very thin forceps which can be inserted into the uterus easily without the need for cervical dilatation. In comparison, the size of standard IUD retrievers ranges between $3.0 \times 2.5 \mathrm{~mm}$ and $3.0 \times 0.7 \mathrm{~mm}$ at the operative end while the size of a standard outpatient hysteroscope with an operating channel is $4 \mathrm{~mm}$ (Gynecare Versascope ${ }^{\mathrm{TM}}$, Ethicon, Wokingham, UK). The ease of intrauterine insertion and the level of discomfort tend to decrease with decreasing size of operating devices passing through the cervical canal. The main risk of using a very thin instrument is the possibility of creating a false passage or causing uterine perforation. This is very unlikely to occur, however, when the procedure is continuously monitored by ultrasound scan. Transabdominal ultrasound monitoring could be difficult in overweight women and those with large fibroids, and in these cases the procedure should be performed with utmost care in order to avoid complications.

The other disadvantage is the difficulty in grasping the body of the IUD with a thin instrument. However, this is rarely required as the threads, the loop at the end of the vertical stem or the arms of the IUD could be grasped without difficulty. The same device is used for hysteroscopic IUD retrieval, which hitherto has been considered to be the most successful procedure for the removal of lost IUDs. ${ }^{9}$

The majority of women in our study reported either no pain or only mild discomfort and the median recorded pain score was 3. Although the level of pain is a critical factor in determining the acceptability and success of any outpatient operating procedure, only a few publications actually report the level of pain that women experience during retrieval of lost IUDs. In a randomised study assessing the value of intrauterine lidocaine for pain relief during removal of lost IUDs using Novak's curette, the mean reported pain scores were 6.4 in the placebo and 5.2 in the treatment group. ${ }^{14}$ We are not aware of any publications reporting pain experienced during hysteroscopic IUD removal. However, a recent randomised study evaluating preoperative oral paracetamol and ibuprofen administration on the pain experienced during diagnostic hysteroscopy reported mean pain scores of 4.52 in the medicated and 4.71 in the non-medicated groups. ${ }^{15}$ These mean pain scores were higher than those in our study. We believe that the narrow diameter of the hysteroscopic grasper and the avoidance of uterine distension are probably the main factors contributing to the low level of pain experienced by our patients.

In the UK, 75\% of women aged 16-49 years use contraception, $8 \%$ of whom opt for either a copper or levonorgestrel-releasing IUD. ${ }^{16}$ There are 15.1 million women aged 16-49 years in the UK, which gives a rough estimate of 1.2 million IUD users. ${ }^{17}$ Missing threads tend to be noted in $5-18 \%$ of cases at the time of IUD removal, ${ }^{4}$ which means that a considerable number of women must experience this complication. Considering that approximately half of such women may require a referral to specialist secondary care services for consideration for hysteroscopy following an unsuccessful attempt to remove the IUD in the primary care or sexual and reproductive health clinic setting, the cost to the National Health Service of managing these cases is considerable. Our method is significantly cheaper than outpatient hysteroscopy as it does not require the use of expensive consumables.

A limitation of our study is that the results are based on a relatively small number of women. The 
success rates should be interpreted with caution and a prospective randomised control trial would be needed to compare the effectiveness of this new method with other established techniques.

In conclusion, we believe that our novel method of ultrasound-guided retrieval of missing IUDs may be a good alternative to the standard techniques currently used for this purpose. Although our initial experience indicates that our approach is more successful and less painful than the alternative methods, these findings should be tested in a prospective randomised trial.

\section{Competing interests None.}

Provenance and peer review Not commissioned; externally peer reviewed.

\section{REFERENCES}

1 Peri N, Graham D, Levine D. Imaging of intrauterine contraceptive devices. J Ultrasound Med 2007;26:1389-1401.

2 Heikinheimo O, Gemzell-Danielsson K. Emerging indications for the levonorgestrel-releasing intrauterine system (LNG-IUS). Acta Obstet Gynecol Scand 2012;91:3-9.

3 Lindh I, Milsom I. The influence of intrauterine contraception on the prevalence and severity of dysmenorrhea: a longitudinal population study. Hum Reprod 2013;28:1953-1960.

4 Prabhakaran S, Chuang A. In-office retrieval of intrauterine contraceptive devices with missing strings. Contraception 2011;83:102-106.

5 Bounds W, Hutt S, Kubba A, et al. Randomised comparative study in 217 women of three disposable plastic IUCD thread retrievers. Br J Obstet Gynaecol 1992;99:915-919.

6 Cetinkaya K, Kumtepe Y, Ingec M. Minimally invasive approach to cases of lost intra-uterine device: a 7-year experience. Eur J Obstet Gynecol Reprod Biol 2011;159:119-121.

7 Marchi NM, Castro S, Hidalgo MM, et al. Management of missing strings in users of intrauterine contraceptives. Contraception 2012;86:354-358.
8 Benacerraf BR, Shipp TD, Bromley B. Three-dimensional ultrasound detection of abnormally located intrauterine contraceptive devices which are a source of pelvic pain and abnormal bleeding. Ultrasound Obstet Gynecol 2009;34:110-115.

9 Konamme SP, Auld BJ, Zaidi J. A novel device for removal of the retained intrauterine device. J Fam Plann Reprod Health Care 2006;32:87-88.

10 Thonneau P, Goulard H, Goyaux N. Risk factors for intrauterine device failure: a review. Contraception 2001;64:33-37.

11 Scanlan KA, Propeck PA, Lee FT Jr. Invasive procedures in the female pelvis: value of transabdominal, endovaginal, and endorectal US guidance. Radiographics 2001;21: 491-506.

12 McCaffery M, Beebe A. Pain: Clinical Manual for Nursing Practice. Baltimore, MD: V.V. Mosby Company, 1993.

13 Mizia K, Ramsay P. The effectiveness and safety of ultrasound-guided removal of a Mirena( $\left.{ }^{\circledR}\right)$ intrauterine system when the strings are not visible and conventional office procedures have failed. Aust N Z J Obstet Gynaecol 2013;53:386-388.

14 Güney M, Oral B, Mungan T. Efficacy of intrauterine lidocaine for removal of a "lost" intrauterine device: a randomized, controlled trial. Obstet Gynecol 2006;108:119-123.

15 Teran-Alonso MJ, Santiago JD, Usandizaga R, et al. Evaluation of pain in office hysteroscopy with prior analgesic medication: a prospective randomized study. Eur J Obstet Gynecol Reprod Biol 2014;178:123-127.

16 Office for National Statistics. Opinions Survey Report No. 41. Contraception and Sexual Health, 2008/09. http://www.ons. gov.uk/ons/rel/lifestyles/contraception-and-sexual-health/ 2008-09/2008-09.pdf [accessed 23 August 2014].

17 Office for National Statistics. Population Estimates for UK, England, Wales, Scotland and Northern Ireland, Mid-2013. http://www.ons.gov.uk/ons/taxonomy/index.html? nscl=Population\#tab-data-tables [accessed 23 August 2014].

\section{Northern Interbranch Group}

Autumn Update meeting on Saturday 10th October 2015 at Fairfield

Manor Hotel, Shipton Road, Skelton, York YO30 1XW

Provisional Programme includes:

- Gynaecological malignancies and ovarian cancer screening.

- Sex after treatment for cancer.

- Contraception for women with PCOS.

- Medico-legal issues in contraception and sexual health services.

- Suitable for FSRH self certification for 4 hours CPD.

Contact for information and application form:-

Angela Walker

Angela.j.walker@btinternet.com 